\title{
Triageempfehlungen für Aufnahme und Verbleib von Kindern auf der Intensivstation bei Influenza-Pandemie
}

\author{
Das folgende Dokument wurde - auf Initiative der Kantone und des Bundesamtes für \\ Gesundheit - erarbeitet durch die Schweizerische Gesellschaft für Intensivmedizin in \\ Zusammenarbeit mit und im Namen der Schweizerischen Gesellschaft für Pädiatrie, der \\ Schweizerischen Gesellschaft für Neonatologie, der pädiatrischen Infektiologiegruppe \\ Schweiz, der Vereinigung der Kantonsärztinnen und Kantonsärzte der Schweiz und \\ dem BAG.
}

Bernhard Frey ${ }^{a, b}$,

Christoph Berger ${ }^{a, c}$,

Christian Kind,

Bernard Vaudauxe

a Erstautoren vom UniversitätsKinderspital Zürich

b Abt. für Intensivmedizin und Neonatologie

c Infektiologie und Spitalhygiene

d Ostschweizer Kinderspital St. Gallen

e Département de Pédiatrie, CHUV Lausanne

Für die Schweizerische Gesellschaft für Intensivmedizin SGI (BF) in Zusammenarbeit mit den Schweizerischen Gesellschaften für Pädiatrie SGP (CK, CB, BV, BF), der Schweizerischen Gesellschaft für Neonatologie SGN (BF, CK), der pädiatrischen Infektiologiegruppe Schweiz (PIGS, CB, BV, $\mathrm{CK})$, der Vereinigung der Kantonsärzte (VKS Dr Chung-Yol Lee; Fribourg) und dem Bundesamt für Gesundheit (BAG, Abteilung Übertragbare Krankheiten MT)

Korrespondenz:

Prof. Dr. med. Bernhard Frey Abteilung für Intensivmedizin und Neonatologie

Prof. Dr. med. Christoph Berger Infektiologie und Spitalhygiene Universitäts-Kinderklinik

CH-8032 Zürich

Tel. 0442667111

bernhard.frey@kispi.uzh.ch

christoph.berger@kispi.uzh.ch
Einführender Kommentar von Dr Chung-Yol Lee, Vizepräsident der Vereinigung der Kantonsärztinnen und Kantonsärzte der Schweiz (VKS)

Wir sind diesmal glimpflich davongekommen. Die Pandemie war viel weniger schwerwiegend, als dies die ersten Erfahrungen in Mexiko und teilweise auch in den Ländern der südlichen Hemisphäre befürchten liessen. Diese relativ milde Pandemie gab uns Gelegenheit, das komplexe Zusammenspiel der Akteure im Falle einer Pandemie - und zum Teil auch im Falle anderer sanitärer Krisen - zu üben und zu evaluieren. Die Schweiz steht in Bezug auf die Pandemievorbereitung im internationalen Vergleich gut da. Die Kommunikation und die Koordination könnte sicher noch optimiert und die Zuständigkeiten zwischen Bund und Kantonen sowie zwischen den verschiedenen Departementen und Ämtern auf allen Ebenen könnten besser definiert werden. Aber auch manche Aspekte der langfristigen Planung bedürfen einer aktiven politischen Entscheidung.

So sind wir nicht nur im Bereich der klinischen Forschung mit Kindern in einem Dilemma. Wir sind es auch im Falle einer schweren Pandemie. Die Anzahl der Intensivpflegeplätze und der Fachkräfte für die Kinder ist stärker limitiert als diejenige für die Erwachsenen. Deren Kapazität lässt sich auch nicht ohne weiteres kurzfristig erhöhen. Dies stellt nicht nur die verantwortlichen Ärzte vor eine ethische Herausforderung. Die ganze Gesellschaft muss sich die Frage stellen, wie weit sie trotz des unsicheren zeitlichen Horizonts mit den eventuellen Investitionen gehen will bzw., welche menschlichen Verluste sie zu akzeptieren bereit ist.

Zu welchem Schluss die Politik und die Gesellschaft auch immer kommen mögen, wichtig ist die Nach-

Eine Influenza-Pandemie kann Kinder (0-16 Jahre) in grossem Ausmass treffen, insbesondere was die Zahl der Erkrankungen betrifft. Die über alle Altersklassen höchste Hospitalisationsrate wird bei Säuglingen ( $<1$ Jahr) erwartet. Zum Vergleich: Bei saisonaler Influ- vollziehbarkeit in der konkreten Situation für die betroffenen Familien, die empfundene (prozessuale) Gerechtigkeit und die rechtzeitige Information darüber, dass gesamtschweizerisch vergleichbare Kriterien der Triage für die Aufnahme und den Verbleib auf der IPS angewendet würden. In diesem Sinne sind die Triageempfehlungen für die Aufnahme und den Verbleib auf der Intensivstation für Kinder im Falle einer Influenza-Pandemie eine unverzichtbare Grundlage zur Planung und Vorbereitung aller Akteure und dies entlastet zumindest teilweise die verantwortlichen Ärzte an der Front

Der Vorstand der Schweizerischen Konferenz der Kantonalen Gesundheitsdirektorinnen und -direktoren (GDK) hat vom Dokument Kenntnis genommen und seinen Mitgliedern eine pragmatische Umsetzung der Experten-Empfehlungen und der daraus zu ziehenden Konsequenzen, jedoch auf regionaler* Ebene empfohlen, welche die bestehenden Kooperationen und Synergien nutzt. Die Diskussionen unter den Behörden und unter den Fachleuten sind damit angestossen. Hoffen wir auf fruchtbare Ergebnisse im Interesse unserer schwächsten Mitglieder der Gesellschaft.

Im Namen der Vereinigung der Kantonsärzte und Kantonsärztinnen der Schweiz möchte ich den Autoren der Empfehlungen danken für die gute Arbeit, die sie mit grossem Engagement trotz des enormen Zeitdrucks geleistet haben. An das Bundesamt für Gesundheit, Abteilung übertragbare Krankheiten, geht unser Dank für die Bereitschaft, unser Anliegen aufzunehmen und das Mandat an die Autoren zu erteilen.

* siehe dazu Situation A, Punkt 3.

enza ist die Hospitalisationsrate von Kindern $<6$ Monate 20-mal höher als bei jungen Erwachsenen, und ebenso hoch wie jene der $>65$-Jährigen. $50 \%$ der hospitalisierten Kinder sind $<6$ Monate, und $80 \%<2$ Jahre alt. Auch wenn die Hospitalisationsrate bei Säuglin- 
gen und Kleinkindern mit Komorbiditäten höher ist, liegen bei zwei Drittel aller wegen Grippekomplikationen hospitalisierten Kinder keine Komorbiditäten oder Risikofaktoren vor [1-4].

Für die Influenza A(H1N1)-Pandemie 2009 hat das BAG im August aufgrund von Hochrechnungen mit 180-300 Hospitalisationen bei Kindern (ca. 100-200 für 0-4-Jährige und ca. 60-120 für 5-14-Jährige), davon 15-25\% mit Intensivpflegebedarf gerechnet. Bei ausserordentlich mildem Verlauf führte die Pandemie zu etwa der Hälfte der vorausgesagten Hospitalisationen und nur einzelne Kinder benötigten Intensivpflege. Dennoch zeigen auch bei dieser milden H1N1Pandemie 2009 die Daten aus Kalifornien, dass Kinder $<18$ Jahre $32 \%$ aller Hospitalisationen ausmachten, oder anders gesagt, dass die Hospitalisationsrate von 12 pro 100000 bei Kindern 4-mal höher war als in der Gesamtpopulation. 26\% der hospitalisierten Kinder benötigten Intensivpflege, ein Drittel von ihnen mechanische Beatmung [5].

\section{Da die Intensivbetten für Neugeborene und Kinder schon im Alltag knapp sind, kommt es bei einer Pandemie unweigerlich zu einer erheblichen Verknappung}

Auch wenn der Verlauf der vergangenen Pandemiewelle sehr viel weniger schwer war, als zu Beginn befürchtet, haben die Vorbereitungen auf eine schwerere Pandemie sehr deutlich eine besondere Vulnerabilität der pädiatrischen stationären Versorgung gezeigt. Erwachsenenspitäler können für den akut erhöhten Bettenbedarf bei einer Pandemie relativ leicht zusätzliche personelle und räumliche Kapazitäten schaffen, indem elektive Spitaleintritte abgesagt, bzw. verschoben werden. Dies ist für pädiatrische Kliniken nur in viel geringerem Ausmass möglich, da der Anteil geplanter Hospitalisationen schon im Normalfall nicht mehr als ein Viertel ausmacht. Diese Tatsache gilt für alle Kinderbettenstationen, ist aber besonders ausgeprägt im Bereich der Intensivbehandlung. Da die Intensivbetten für Neugeborene und Kinder schon im Alltag knapp sind, kommt es bei einer weniger mild verlaufenden Pandemie unweigerlich zu einer erheblichen Verknappung.

Im Sommer 2009 wurde die Kapazität der Intensivpflege-Stationen für Erwachsene und Kinder in der Schweiz mittels einer Erhebung des BAG in den Kantonen ermittelt. Diese Erhebung ergab zusammengefasst rund 850 Intensivpflegeplätze für Erwachsene, verteilt auf ca. 80 Zentren, mit schätzungsweise 350-700 Respiratoren. Pädiatrische Intensivpflegeplätze sind es ca. 90-100, verteilt auf die 8 Zentren UKBB (BL+BS) Basel, Inselspital Bern, KS Graubünden (Chur), HUG Genève, CHUV Lausanne, KS Luzern,
Ostschweizer Kinderspital St. Gallen und Kinderspital Zürich, mit insgesamt ca. 40-70 Respiratoren (ohne Neonatologie).

Ausgehend vom Pandemieplan der Schweiz [6], dem Positionspapier der Schweizerischen Gesellschaft für Intensivmedizin [7], Angaben in der Literatur [8] und Überlegungen zur Triage bei Kindern [9] wurden die nachfolgenden Empfehlungen erarbeitet.

Während einer pandemischen Grippewelle wird zwischen zwei Situationen unterschieden: A: eine Situation zu Beginn und am Ende der Pandemiewelle, während der in der Schweiz noch genügend Intensivpflegeplätze und Personal vorhanden sind, und B: eine Situation am Gipfel der Pandemiewelle, in der praktisch alle IPS-Plätze der Schweiz belegt und/oder wegen grösserer Personalausfälle nicht verfügbar sind.

\section{Situation A: Freie Plätze / \\ Personal noch vorhanden}

Es muss alles daran gesetzt werden, dass diejenigen Patienten, die einer Intensivtherapie bedürfen, diese auch erhalten. Dazu müssen die folgenden Massnahmen in Betracht gezogen und vorbereitet werden:

1. Ressourcenerhöhung: Zeitliche Verschiebung elektiver Operationen und Hospitalisationen. Evtl. Beschaffung zusätzlicher Beatmungsgeräte, Rekrutierung (z. B. Pensionierte) und Schulung von zusätzlichem Personal. Vorübergehende Erhöhung der Arbeitszeit auf das höchstmögliche Niveau (z. B. durch Ferienverschiebung usw.).

2. Ressourcenausdünnung: Durch Reduktion der Behandlungs- und Pflegeintensität auf ein tieferes Niveau kann mit vorhandenen Ressourcen eine grössere Zahl Patienten behandelt werden.

3. Zuweisungen von Patienten: An Standorten mit getrennten pädiatrischen und neonatologischen Intensivstationen (Universitätsspitäler) muss eine enge Zusammenarbeit zur optimalen Ausnützung der Ressourcen erfolgen. Die Verlegungen von Kindern aus Kantonen ohne Kinder-IPS erfolgen zunächst gemäss üblichem Prozedere bei interkantonalen Verlegungen, punktuell bestehen dafür interkantonale Vereinbarungen. Als 2. Schritt (bei ersten Engpässen) kann eine Verlegung bzw. Einweisung von Kindern über $30 \mathrm{~kg}$ auf die Erwachsenen-Intensivpflege-Stationen der Schweizer Spitäler, die über eine solche verfügen, erfolgen. In einem 3. Schritt (zunehmende Engpässe = Übergang in Situation B, siehe unten) soll eine national koordinierte Zuweisung von Kinder-IPS-Plätzen innerhalb der Schweiz erfolgen. Dazu muss ein laufend (z.B. täglich) aktualisiertes Informationssystem zur Verfügung stehen, mit dem freie IPSPlätze in der Schweiz für Kinder und Erwachsene abgerufen werden können. Ein mögliches solches Instrument könnte die webbasierte Informatikplattform IES (Informations- und Einsatz-System des Koordinierten Sanitätsdienstes-KSD) sein. 
Um die entsprechenden Voraussetzungen für solche Massnahmen zu schaffen, kann das BAG gegenüber den kantonalen Behörden, d.h. den Kantonsärztlichen Diensten und der Gesundheitsdirektorenkonferenz GDK, eine beratende Funktion einnehmen.

Ethik: In den einzelnen Intensivstationen wird der dort übliche Weg der ethischen Entscheidungsfindung beschritten.

\section{Situation B: Alle IPS-Plätze der Schweiz belegt und/oder Personalausfälle}

Sobald nahezu alle IPS-Plätze der Schweiz belegt sind, muss ein schweizweit einheitlicher Triageprozess (=Rationierung) einsetzen. Bei ungenügenden Ressourcen trotz optimalem Einsatz der unter A angegebenen Massnahmen muss zunächst die limitierende Ressource (in der Regel die maschinelle Beatmung) rationiert werden. Mit dem Ziel, möglichst viele Patienten am Leben zu erhalten, muss einzelnen Patienten, deren kurzfristiges Überleben weniger wahrscheinlich ist, die lebenserhaltende Therapie vorenthalten werden. Die Rationierung betrifft in dieser Situation alle Patientenkategorien (Kinder und Erwachsene). Dieses Dokument beschränkt sich auf die Kinder (0-16 Jahre).

\section{Kriterien für Aufnahme auf die Intensivstation für Kinder}

Die Einteilung in drei Kategorien soll für die Triage eine Hilfe sein. Es ist aber nicht möglich, die Triage anhand starrer Kriterien (z. B. Zahl, Score) durchzuführen*. Jeder Triageentscheid sollte möglichst nicht durch eine Einzelperson, sondern durch eine spitalinterne, designierte Gruppe («Rationierungskommission») - bestehend aus Ärzten und Pflegenden mit langjähriger Erfahrung in pädiatrischer Intensivmedizin - gefällt werden. An Spitälern mit pädiatrischer IPS wird eine solche Kommission durch die Spitalleitung vor Beginn der Pandemie in der Schweiz eingesetzt. In Spitälern, in denen dies nicht praktikabel ist, muss zumindest eine Person bestimmt werden, welche die strikte Einhaltung der Triagerichtlinien durch die Entscheidungsträger gewährleistet. Die Kommission fällt den Triageentscheid unabhängig vom Wohnkanton des Patienten, und berücksichtigt dabei die folgenden Kategorien zur Festlegung der IPS-Priorität (s. Tab. 1):

\section{Kategorie 1}

Schweres Organversagen mit guter Prognose bei Behandlung auf der IPS (meist Einzel-Organ-Versagen

Vorhandensein chronischer Erkrankungen.

\section{Tabelle 1}

Situation B: alle IPS-Plätze der Schweiz belegt und/oder Personalausfälle.

\begin{tabular}{|c|c|c|c|c|}
\hline Kategorie/ Priorität & Beschreibung & Beispiele & Parameter für Organversagen & Prozedere \\
\hline $1 /$ hoch & $\begin{array}{l}\text { Schweres Organversagen mit } \\
\text { guter Prognose bei Behandlung } \\
\text { auf IPS (meist Einzel-Organ- } \\
\text { Versagen ohne schwere Grund- } \\
\text { krankheit) }\end{array}$ & \multirow{2}{*}{$\begin{array}{l}\text { - Respiratorisches Versagen (z. B. durch } \\
\text { Influenza-Pneumonie, bakterielle } \\
\text { Pneumonie oder RSV-Bronchiolitis), } \\
\text { obere Atemwegsobstruktion, Asthma, } \\
\text { Atemnotsyndrom des Neugeborenen } \\
\text { - Sepsis/neonatale Sepsis } \\
\text { - pulmonale Hypertonie } \\
\text { des Neugeborenen } \\
\text { - Gastroenteritis/Dehydratation } \\
\text { - Omphalozele/ Gastroschisis } \\
\text { - Schädel-Hirn-Trauma } \\
\text { - Verbrennung < } 80 \% \text { der Körperober- } \\
\text { fläche (Überlebenschance bei } 80 \% \text { - } \\
\text { Verbrennung: ca. } 50 \% \text { ) } \\
\text { - Diabetische Ketoacidose } \\
\text { - Herzrhythmusstörung }\end{array}$} & $\begin{array}{l}\text { a) Atemversagen: } \mathrm{SaO}_{2}<80 \% \\
\text { mit } \mathrm{FiO}_{2}>60 \%\left(=2 \mathrm{I} / \mathrm{min} . \mathrm{O}_{2}\right. \\
\text { per Nasenprongs bei Säuglingen, } \\
\text { resp. } 5 \mathrm{I} / \text { min per Maske), rezi- } \\
\text { div. Apnöen; } \\
\text { b) Kreislaufversagen: fehlendes } \\
\text { Ansprechen auf Volumentherapie; } \\
\text { c) Enzephalopathie: } \mathrm{GCS}<8 \text { und/ } \\
\text { oder Hirndruckzeichen }\end{array}$ & $\begin{array}{l}\text { Intensiv- } \\
\text { pflege } \rightarrow \\
\text { tägliches } \\
\text { Reassess- } \\
\text { ment: } \\
\text { Kat. 2/3? }\end{array}$ \\
\hline $2 /$ mittel & $\begin{array}{l}\text { Notwendigkeit der intensiven } \\
\text { Überwachung bei potentiellem } \\
\text { Organversagen } \\
\text { (Atmung, Kreislauf; ZNS) }\end{array}$ & & $\begin{array}{l}\text { a) Atemversagen: } \mathrm{SaO}_{2}>80 \% \\
\text { mit FiO } \mathrm{F}_{2}<60 \%\left(=2 \mathrm{I} / \mathrm{min} . \mathrm{O}_{2}\right. \\
\text { per Nasenprongs bei Säuglin- } \\
\text { gen, resp. } 5 \mathrm{I} / \mathrm{min} \text { per Maske), } \\
\text { keine rezid. Apnöen; } \\
\text { b) Kreislaufversagen mit gutem } \\
\text { Ansprechen auf Volumentherapie; } \\
\text { c) Enzephalopathie: GCS }>8 \text { ohne } \\
\text { Hirndruckzeichen }\end{array}$ & $\begin{array}{l}\text { Intensive } \\
\text { Über- } \\
\text { wachung } \\
\text { ausserhalb } \\
\text { der IPS } \rightarrow \\
\text { tägliches } \\
\text { Reassess- } \\
\text { ment: } \\
\text { Kat. } 1 /(3) \text { ? }\end{array}$ \\
\hline 3 / niedrig & $\begin{array}{l}\text { Schlechte kurz- bis mittelfristige } \\
\text { Prognose, trotz Behandlung } \\
\text { auf IPS: } \\
\text { a) Schweres Organversagen bei } \\
\text { vorbestehender schwerer } \\
\text { Grundkrankheit. } \\
\text { b) Krankheit mit per se infauster } \\
\text { Prognose }\end{array}$ & $\begin{array}{l}\text { a) Wie bei Kat. } 1 \text { und 2, mit zusätzlicher } \\
\text { Komorbidität wie: } \\
\text { - extreme Frühgeburtlichkeit } \\
\text { - schwerer Herzfehler (wie hypo- } \\
\text { plastisches Links-Herz-Syndrom) } \\
\text { - vorbestehende pulmonale Hypertonie } \\
\text { - chronische Lungenerkrankung mit } \\
\text { zusätzl. } \text { O }_{2} \text {-Bedarf } \\
\text { - neurodegenerative Erkrankung } \\
\text { - schwerer, kombinierter Immundefekt, } \\
\text { Leukämie oder Lymphom im Rezidiv } \\
\text { - Kardiomyopathie oder Myokarditis } \\
\text { - Leberversagen } \\
\text { b) Zwerchfellhernie mit schwerer Oxyge- } \\
\text { nationsstörung (Oxygenationsindex } \\
\text { >40), Missbildungssyndrom mit Betei- } \\
\text { ligung vitaler Organe, Verbrennung } \\
>80 \% \text { der Körperoberfläche }\end{array}$ & Wie bei Kat. 1 & $\begin{array}{l}\text { Palliativ } \\
\text { pflege } \rightarrow \\
\text { tägliches } \\
\text { Reassess- } \\
\text { ment: } \\
\text { Kat. } 1 /(2) ?\end{array}$ \\
\hline
\end{tabular}


ohne schwere Grundkrankheit). Eine Behandlung nur mit CPAP in einer pädiatrischen Klinik ohne pädiatrische Intensivstation würde die Prognose deutlich verschlechtern. Kategorie 1 hat höchste Priorität.

\section{Kategorie 2}

Patienten, die lediglich eine intensive Überwachung (evtl. CPAP) brauchen. Viele Patienten mit Organversagen können ausserhalb der IPS überwacht und betreut werden, solange die Kriterien für ein schweres Organversagen nicht erfüllt sind. Die Hospitalisation von Kindern der Kategorie 2 soll auch in pädiatrischen Kliniken ohne pädiatrische Intensivstation oder auf Erwachsenen-IMC (Intermediate Care; technische Voraussetzungen: Befeuchtung, Absaugen, $\mathrm{O}_{2}$-Therapie, Monitoring) erfolgen. Mittlere Priorität.

\section{Kategorie 3}

Patienten mit schwerem Organversagen und zusätzlich einer vorbestehenden schweren Grundkrankheit mit schlechter kurz- bis mittelfristiger Prognose sowie Patienten mit infauster Prognose. In Zeiten genügender Ressourcen werden diese Patienten gelegentlich für einen Therapieversuch auf die IPS aufgenommen. Wenn im Falle einer Pandemie die Nachfrage nach IPS-Plätzen das Angebot übersteigt, haben diese Patienten die tiefste Priorität für eine intensivmedizinische Behandlung. Im Falle einer Nicht-Aufnahme auf die IPS müssen sie eine korrekte palliative Behandlung erhalten.

\section{Kriterien für Verbleib auf der Intensivstation}

Kontinuierliches (z. B. tägliches bzw. dem Bedarf angepasst auch häufigeres) Reassessment der Patienten anhand der oben genannten Kategorien zur Festlegung der IPS-Behandlungspriorität. Fällt der Patient immer noch in die gleiche Kategorie? Die Rationierungskommission muss prüfen, ob ein Verbleib auf der IPS gerechtfertigt ist. Dies erfolgt immer auch unter Berücksichtigung der aktuellen pandemischen Lage bzw. der schweizweiten IPS-Belegung, sowie dem ethischen Prinzip des «Nicht-Schadens». Dies bedeutet, dass die Intensivtherapie bei einem Patienten mit ungünstigerer Prognose nur abgebrochen wird, wenn dies zugunsten eines anderen Patienten mit eindeutig besseren Aussichten geschieht. Gut abschätzen lässt sich die Prognose bei: hypoxisch-ischämischer Encephalopathie, Schädel-Hirn-Trauma, Sepsis, Atemnotsyndrom des Neugeborenen, obere Atemwegsobstruktion, Diabetische Ketoazidose.

Ethik: Die zugrunde liegenden ethischen Prinzipien wurden auch im Influenza-Pandemieplan Schweiz 2009 diskutiert (Teil III, S. 234-239). Das Leben der Menschen ist das höchste zu schützende Gut, weil alle anderen Güter von ihm abhängen. Dabei gelten u.a. folgende ethische Werte: Solidarität, Gerechtigkeit, Gleichbehandlung, Fairness, individuelle Freiheit, Privatsphäre, Verhältnismässigkeit, ferner: Transparenz, Anpassungsfähigkeit, Begründetheit und Konsistenz der Entscheide, sowie Vertrauen. Es gelten für alle Pa- tienten (mit oder ohne Influenza) die gleichen Triagekriterien. Der Triageentscheid ist unabhängig von Alter, Geschlecht, Wohnkanton, Nationalität, religiöser Zugehörigkeit, sozialem Status oder vorliegender chronischer Behinderung. Der Entscheid soll im Wesentlichen von der Wahrscheinlichkeit abhängen, mit Hilfe der in Frage stehenden Behandlung die akute Erkrankung zu überleben, und nicht von der mittel- oder längerfristigen Lebenserwartung. Eine Ausnahme bilden Patienten mit einer Komorbidität, die kurzfristig eine schlechte bis infauste Prognose aufweist. In einer Situation mit zu knappen Ressourcen - und mit dem Ziel, möglichst viele Menschen am Leben zu erhalten - sollen diese Patienten nur palliativ behandelt werden.

Kommunikation: Die Triagekriterien müssen den behandelnden Ärzten, der Pflege, den Betroffenen und Angehörigen wie auch den Behörden und der Bevölkerung verständlich kommuniziert werden. Eine offene, ehrliche und zweckmässige Kommunikation trägt dazu bei, ein Klima des Vertrauens und der Solidarität zu schaffen. Ausserdem erlauben es TriageRichtlinien Ärztinnen und Ärzten, die während der Pandemie Entscheide zu treffen haben, diese anhand objektiver Kriterien fällen zu können, sowie allfällige negative Entscheide gegenüber Angehörigen/Eltern auch sachlich begründen zu können, was den Druck auf die entscheidungstragenden Medizinal- und Pflegepersonen erleichtern dürfte.

\section{Literatur}

1 Neuzil KM, Mellen BG, Wright PF, Mitchel EF, Griffin MR, Griffin JR. The effect of influenza on hospitalizations, outpatient visits, and courses of antibiotics in children. N Engl J Med. 2000;342:225-31.

2 Chiu SS, Lau YL, Chan KH, Wong WH, Peiris JS. Influenza-related hospitalizations among children in Hong Kong. N Engl J Med. 2002;347:2097-103.

3 Peltola V, Ziegler T, Ruuskanen O. Influenza A and $B$ virus infections in children. Clin Infect Dis. 2003;36:299-305.

4 Iskander M, Booy R, Lambert S. The burden of influenza in children. Curr Opin Infect Dis. 2007;20:259-63.

5 Louie JK, Acosta M, Winter K et al. Factors associated with death or hospitalization due to pandemic 2009 influenza $\mathrm{A}(\mathrm{H} 1 \mathrm{~N} 1)$ infection in California. Jama. 2009;302:1896-902.

6 Bundesamt für Gesundheit. Influenza-Pandemieplan Schweiz: www.bag.admin.ch/influenza/01120/ 01134/03058/index.html, 2009.

7 Schweizerische Gesellschaft für Intensivmedizin (SGI) Positiionspapier: Intensivmedizinische Aspekte einer Influenzapandemie In: SGI-SSMI-SSICM Guidelines www.sgi-ssmi.ch/sgi-richtlinien0.html, 2007.

8 Christian MD, Hawryluck L, Wax RS et al. Development of a triage protocol for critical care during an influenza pandemic. Cmaj. 2006;175:1377-81.

9 Kind C. Pandemieplan des Ostschweizer Kinderspitals. Triage bei Pandemie. Auf Anfrage beim Autor erhältlich. 